\title{
LOAD BALANCING AND ENERGY EFFICIENCY IN WSN BY CLUSTER JOINING METHOD
}

\author{
Dr Syeda Gauhar Fatima \\ Professor ECE dept, Deccan College of Engineering and Technology, \\ Darussalam, Hyderabad, India \\ Syeda Kausar Fatima
}

Associate Professor, Shadan College of Engineering and Technology, Hyderabad, India

Dr K.Anitha Sheela

Professor ECE dept, JNTUH, Kukatpally, Hyderabad, India

Syed Shaker Hussaini

Student ECE dept, Deccan College of Engineering and Technology,

Darussalam, Hyderabad, India

\begin{abstract}
ABTRACT
In any WSN life of network is depending on life of sensor node. Thus, proper load balancing is very useful for improving life of network. The tree-based routing protocols like GSTEB used dynamic tree structures for routing without any formation of collections. In cases of larger networks, the scheme is not always feasible. In this proposed work cluster-based routing method is used. Cluster head is selected such that it should be close to the base station and should have maximum residential energy than other nodes selected for cluster formation. Size of cluster is controlled by using locationbased cluster joining method such that nodes selects their nearest collection head based on the signal strength from cluster head and distance between node and cluster head. Nodes connect to head having the highest signal strength and closest to the base station, this minimizes size of cluster and reduces extra energy consumption. In addition to this cluster formation process starts only after availability of data due to an event. So proposed protocol performs better than existing tree based protocols like GSTEB in terms of energy efficiency.
\end{abstract}

Keywords: Wireless Sensor Network, Energy efficiency, load balancing, clustering, routing.

Cite this Article: Dr Syeda Gauhar Fatima, Syeda Kausar Fatima, Dr K.Anitha Sheela and Syed Shaker Hussaini, Load Balancing and Energy Efficiency in WSN by Cluster Joining Method, International Journal of Advanced Research in Engineering and Technology, 10(2), 2019, pp. 124-134.

http://iaeme.com/Home/issue/IJARET?Volume $=10 \&$ Issue $=2$ 


\section{INTRODUCTION}

Wireless sensor network is made up of number of sensor nodes mostly deployed in large quantity due to their energy limitation. The way by using which data gathering is performed is very important to any WSN application. Information collection and routing based on clusters are widely used method because it provides better energy efficiency .Load balancing clusters are formed by considering their position and considering their residential energy. One node is selected as a head node which collects all the data from group members, aggregates them and finally forwards it by using routing algorithm .during each round role of cluster head changes it produces better load balancing. Clustering avoids communication of same data from multiple path and minimizes energy consumption. With energy other factors related to the QoS must be considered for better performance of the system .One of the important QoS limitation is delay .End to End delay of WSN should be minimum[ 1 ].It is possible to achieve this objective by using cluster based routing. We can save energy either at network layer by using proper routing algorithm and at MAC layer by using proper MAC protocol .By selecting proper routing protocol and proper MAC protocol we can also control delay in any WSN application. Delay should be important design issue in particular types of applications such as military applications ,earthquake monitoring[ 3 ] . Clustering is useful to reduce communication distance of nodes to reduce energy required for communication of data[4].In any WSN hierarchical routing mechanism is useful to avoid redundant data communication. We can also conserve energy by adopting proper

Duty cycle by keeping nodes active which have data to sense and to transmit and keeping all remaining nodes to sleep state who don't have any data to transmit .Combination of these two methods can network life time and overall performance[6].

Use of mobile sink is good idea to balance network load properly in order to improve lifespan of system. Tree based routing is also used for load balancing. Work GSTEB [09] has provided this type of protocol and in work [10] has provided improved version of this protocol.

In proposed work cluster head is selected close to the base station. In addition we have tried to control unnecessary size of cluster because redundant extra size of cluster consumes extra energy. To handle this issue during formation of group member nodes selects their cluster head based on distance and signal strength coming from cluster heads. TDMA is also used as medium access protocol to improve performance.

If cluster formation starts only after availability of data due to event, this minimizes power required to construct redundant cluster formation which doesn't have any information[11]. We used this method for more energy efficiency .

Section two is provided with related work in the same area. Third section provides architecture of proposed work and detailed explanation of protocol. Fourth section provides performance analysis and comparison graph with existing system.

\section{RELETED WORK}

In many WSN applications with life of network some quality of service must be provided. Some research work on WSN is only considering lifetime of network but QoS should be considered mostly in case of applications such as military surveillance and battleground monitoring in case of these applications end to end delay should be minimum .Author Min Yao etal. of work[ 1 ]has tried to handle this issue. They have proposed model which can consider both problem of lifetime of network and QoS requirements mostly the delay between source and sink and data loss during communication process. Their results show that the work improves network lifetime with maintaining QoS requirements [ 1 ]. 
Periodically turning off the radio of sensor node is good solution to energy consumption but this may increase end to end delay in the WSN .Here number of packets to the sensor node are controlled to minimize energy consumption and end to end delay of the system .author Yun Li etal. Of [2] used dynamic programming approach to create proper communication policy to improve performance of the system their simulation results shows that their work is $50 \%$ better than existing IEEE802.15.4 work.

In case of LEACH when node has no data in its TDMA slot its TDMA slots will be wasted .Work[3] op leach handles this by conveying TDMA slots of nodes having no data to send by conveying its TDMA slot to next node to avoid wasting of TDMA slot. This work is useful for both energy efficiency and delay minimization. [ 3 ].

Another important work is provided in paper [4] This work used concept of clusters similar to LEACH for routing and load balancing but they have also provided solution on security threats on cluster heads such as gray hole attack. Their results shows that their system is better than LEACH because their proposed work also addresses problem of gray hole attack. This is the attack in which any malicious sensor device can send fake data to the cluster head to engage sensor node in an unnecessary activity. This will increase energy consumption and delay in the system [4].

$\mathrm{t}$ is very important to solve problem of global clock synchronization .author Supantha Das etal. Of work [5] tried this by avoiding use of clock synchronization instead they have provided new technique of coloring. Network is organized in tree structure .Parallel communication is achieved for better performance. Their results shows that their system works better as compared to other related work in terms of both energy consumption and End to End delay .Instead of using global time they have used local time synchronization .Inter level and intra level coloring method is used to improve parallelism and to avoid impact [5]. Combined approach of energy efficient scheduling and hierarchical routing is provided by author of work [6].First they apply power aware scheduling by activating only those nodes involved in data processing and communication and remaining nodes are in sleep mode.After that hierarchical routing is used for power saving. This combined approach works better in terms of energy efficiency. Their simulation results shows that their work is better than existing systems such as LEACH, PASC, M GEAR systems. [6].

Use of mobile sink is good idea for energy efficient load balancing ,such type of work is presented by author Wen-Hwa Liao etal. in paper [7]. They have categorized nodes in to rendezvous nodes and non-rendezvous nodes .Here only rendezvous nodes can communicate with the mobile sink and non-rendezvous nodes can send their data to the rendezvous nodes .Here mobile sink can travel to the rendezvous nodes in the area and collect desired data in the energy efficient way and this works well for improving network life time by reducing distance between sink and rendezvous nodes[7].

In any WSN when more than one node tries to transmit data at the same time using single communication medium then it will create contention for accessing medium and impact of data .If impact is more than particular limit this will consume extra energy and delay in the system due to recommunication of data and reduces QoS requirements of the system .If contention is due to correlated traffic there is possibility of more contention so author Ashutosh Bhatia etal.of [ 8 ] tried to solve this problem .TDMA is good solution for this but according to [8 ]if TDMA schedule is not generated within short periods of time then it will be useless because correlated contention presents only for short duration of time so author has proposed RD-TDMA algorithm which generates feasible TDMA schedule in very short period of time[8 ] .

Tree based routing is very useful for load balancing. Work [09] by author Zhao Han etal.has used tree based routing for load balancing . dynamically created tree is used for communication of data from sensor node to root. Here tree will be created in minimum time and load is properly 
balanced using tree structure .Work [09]is best but problem with [09] is that load is not distributed in node centric way and base station is involved in tree construction so lot of data communication between base station and remaining nodes will increase delay. To solve this problem work number [10] by M.Sengaliappanetal. has provided improved version of this tree based routing.Author has used cluster based routing for node centric load balancing. In this work base station is very less involved in actual data communication so delay in the system is also minimized[10]. Cluster formation only after event detection provides better performing network because it reduces unnecessary energy consumption.Work [11]is same type of work .They have implemented their work using MATLAB simulator. Their results are better than existing cluster based applications SCHS and EESH.

Use of multiple sink is another way for load balancing and for timely delivery of data,author of work [12] has tried to solve problem of load balancing by using multiple sinks instead of using single sink. They have provided efficient task allocation method in large scale WSN.Their results are better in terms of packet delivery ratio, speed of data aggregation and time required for processing and communication of data. Multiple sinks used are mobile in nature it improves total performance of the system [12].

Cross layer optimization is better way for energy efficiency and load balancing, work[13]is based on this approach. With collecting they have also used cross layer approach to improve life of the network. This work focuses on all three main layers at which we can control power consumption. This layers are physical layer, MAC layer and routing layer .At physical layer they control communication power. At Medium Access Layer they have used S-MAC duty cycling and at network layer they have implemented energy efficient routing method This network has capability of dynamically reconfiguration based on current condition of the network and network topology change. [13].Similar work is provided by author of [14]. Node sleeping is good for energy efficiency but it increases unnecessary end to end delay in the WSN ,so this author has tried to address this issue by adopting new routing method.Wake up rate is provided with each node , using this wake up rate relay node is selected for routing purpose. It is very useful to control delay in the network. This works well than heuristic methods[14].Optimal woke up rate is provided because large wake up rate increases extra energy consumption. Data packets are created and forwarded only after detection of event and this avoids unnecessary energy consumption of the sensor node.

Node close to the base station or sink will consume energy in fast way. This will create holes in the network and this will result in early death of network. To avoid this author of work[15]provided concept of multiple sink. When multiple sinks are used then total load is distributed properly. Instead of creating single routing tree they have created multiple routing trees towards each sink in the network. This reduces extra time and energy consumption[15].

Author of work [16] considers both these performance aspects and provided tradeoff between End to End delay and energy consumption .New algorithms are provided for inter cluster routing and for intra cluster routing. Two functions are created for both for optimization purpose .Clusters are created in distributed way to evenly distribute network load on all the cluster nodes .Cluster heads are selected by considering both factors energy and delay. They have provided results in two forms theoretical and simulation, both results are same up to some percentage [16].

Congestion free and free wireless sensor network provides better output in terms of energy ,delay and minimum packet drop. Author of work[17] has considered and implemented this issue by constructing congestion free routing tree. They have proposed CATopology i.e. Congestion Avoidance Topology by using two methods known as K-Map and K-Graph. Routing on this multi hop routing tree dose not allows more than one packets to reach at the same point in the network at the same time period. This improves energy efficiency, minimizes 
end to end delay and also minimizes number of packet drops in the network. They have simulated their work by using OPNET network simulator . Their results are better than similar work[17]. Author of work[18] provides method of cluster formation in distributed way. They have tried to distribute network load on each and every node in the network. They have addressed problem of scalability and energy efficiency. TDMA is also used for performance improvement and sleep wakeup method is used for more energy saving .For selecting cluster head both distance and residential energy of node is considered [18].

Work [19] has provided generic algorithm for load balancing purpose .They have used different strategy for generation of initial population to generate chromosomes which are valid. For proper load balancing they have used fitness function to balance network load properly. Each cluster member node can communicate with cluster head node by using single hope or using multi hope communication. According to the requirement. If node is very close to the head it can communicate directly and if node is not close to cluster head then it can use malty hope path for communication purpose. The work is better in terms of energy efficiency[19].

GSTEB is a tree based routing protocol. In GSTEB routing tree is generated dynamically in each round. Here round means time from sensing data up to the time at which data is delivered to the base station. Work[20]is based on improvement in tree based routing protocol .Author of work[20] has added concept of fuzzy rules for creation of clusters and for selecting proper routing path. Use of fuzzy rule has shown better performance in terms of energy efficiency and delay minimization in the WSN. Network simulator 2 is used for implementation purpose. Results show that system performs better than existing HEED and GSTEB protocols [20].

Author of [21] has divided sensing area into four parts. Each part is termed as quadrant. Parent or relay node is selected by considering distance between nodes and residential energy of node ,this makes better relay node selection for minimum energy consumption. TDMA is used for resource allocation purpose. Each cluster head performs TDMA schedule for each node inside the cluster and broad cast this schedule to all cluster members. All cluster members follows this TDMA schedule for data communication purpose. They have implemented their work by using MATLAB simulator and results are better than existing NRLM and LEACH protocols [21].

\section{IMPLEMENTATIONDETAILS}

We assumed system model similar to the GSTEB. Sensor nodes are deployed in square area randomly. Only single base station is provided and which is far away from the sensing area .Any node can communicate directly with the base station if it is closer to it by changing its current remaining energy. All sensor nodes have their fixed position. That is it is assumed that they cannot change their position . Base station is also stationary but it is assumed that it is no energy constrained 


\subsection{System architecture of proposed work}

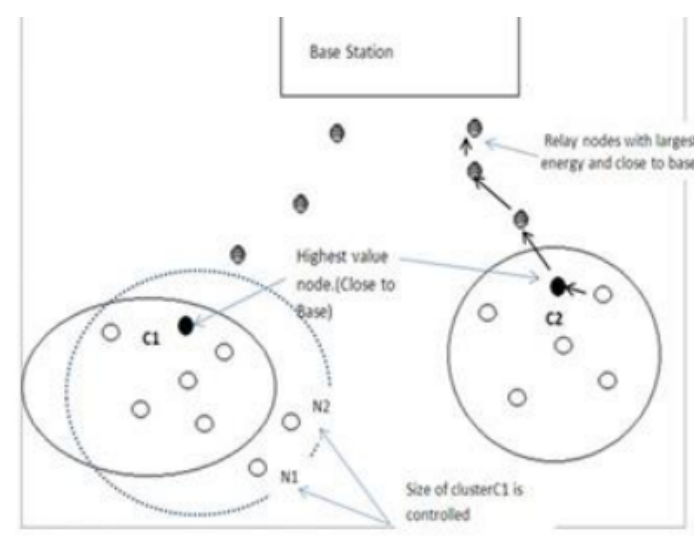

Figure 1 Closest cluster head to the base station with maximum energy level.

Above figure 1 shows architecture for proposed protocol. Proposed work has four phases which are described in following section.

\subsection{Phases involved in proposed approach}

\subsubsection{Phase 1. Cluster formation phase}

Cluster formation process starts with a node which listens some event related to the availability of data, we can say it as a seed node. Now other nodes which are in the communication range of seed node accept the cluster formation request from seed node and joins this node for cluster formation process .If two nodes listen event at the same time then node having maximum energy is selected as seed node. If two nodes listen event at the same time but at large distance from each other such that they are not within communication range of each other in this case both are considered as seeds for two different clusters it is shown in fig 2.

Base is indicated by green circle. Here in the area two seed nodes are selected and they are indicated by brown colored circles. Communication range of each seed is considered as 250 meter, here both seeds are not within communication range of each other, so both are considered as two separate seeds for two separate cluster formation. Violet color nodes are within communication range of seed 1 and magenta color nodes are within communication range of seed2.

Algorithm for seed node selection

Step 1: If seed1 and seed2 are within communication range of each other and energy of seed 1 is greater than seed 2 then seed 1 is selected as seed node

Step2: If seed 1 and seed 2 are not within communication range of each other then both are considered as separate seed nodes for two deferent cluster formations

Step3: Each selected seed sends cluster formation request to its neighbors who are within its communication rage.

Step4: Nodes within communication range of seed are selected for cluster formation process

We can calculate energy level of any node by using following equation.

$$
E(i)=\text { Residual Energy }(i) / \alpha
$$

If there are $\mathrm{N}$ number of nodes then $\mathrm{i}$ indicates $\mathrm{i}$ th node. $\mathrm{E}=$ Current energy level. $\alpha=$ smallest energy unit. 


\subsubsection{Phase 2. Cluster Head Selection Phase}

In this phase cluster head is selected based on two criteria's. first is distance between base station and candidate nodes for cluster head selection and second criteria is energy level of node. First criteria selects cluster head closest to the base station . Selecting closest cluster head to the base station minimizes energy consumption for communication of data .This is useful because energy required for communication of data is higher than energy required for receiving the data and for processing the data. Based on these two criteria values for each node in the cluster is calculated. Node having maximum value is selected as cluster head.
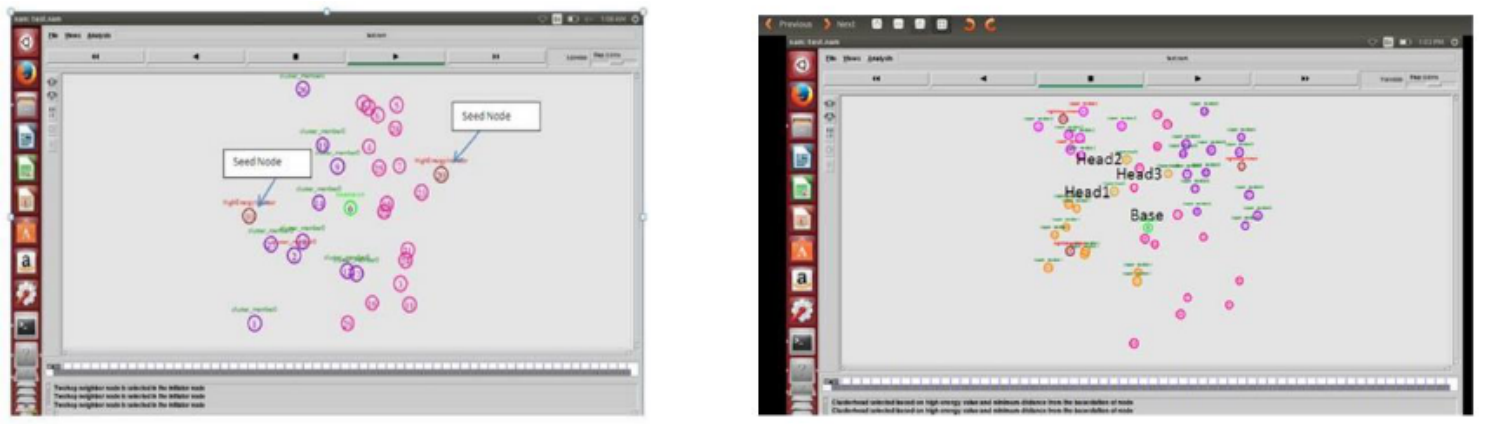

Figure 3 Cluster head selection

As shown in the fig 3 base stations are indicated by green color. Three cluster heads are selected for three different cluster formations. Cluster heads are indicated by light orange color. Members of cluster for head 1 are shown by dark orange color and members of cluster for head 2 are shown by magenta color and members of cluster for head 3 are shown by dark violet color. Important point here is to note that all these cluster heads are close to the base station and having high energy.

Algorithm for cluster head selection phase

Step1: For all nodes selected by each seed distance between nodes and base station is calculated.

Step2: Node closest to the base station and having highest energy is selected as cluster head.

Step3: If there are two nodes having same highest energy and closest to the base station then cluster head is selected

randomly

We know that the length of line in between any points $\mathrm{P}$ and $\mathrm{Q}$ is known as Euclidean distance [12].We have used same formula to calculate distance between sensor nodes If $p$ and $\mathrm{q}$ are two nodes then we can calculate distance between them as follow [12] .

$$
D(p, q)=\sqrt{(q 1-p 1) 2+(q 2-p 2) 2}
$$

\subsubsection{Phase 3. Location based cluster joining and controlling cluster size.}

After selecting cluster head which is close to the base station and having maximum energy level .Now it is time to control size of cluster .Because unnecessary extra size of cluster will increases energy consumption .In this phase selected cluster head sends request message to join to the remaining nodes in the cluster. Based on strength of message coming from all the nearest head and based on distance between member nodes and $\mathrm{CH}$, a node selects their cluster head. In this way node selects $\mathrm{CH}$ based on physical position that is distance and using actual performance of cluster head i.e. its signal strength. This controls size of cluster in effective way .According to the architecture shown in figure 1 sensor node $\mathrm{N} 1$ and node $\mathrm{N} 2$ will not becoming part of cluster of head $\mathrm{C} 1$ to control size of cluster. 


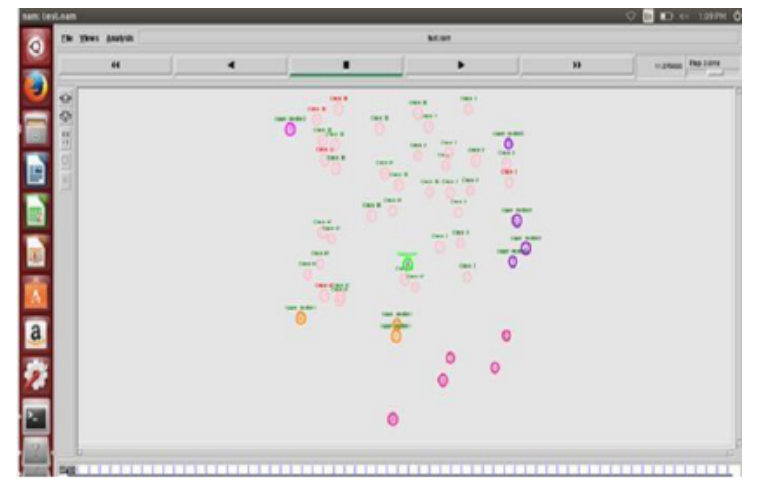

Figure 4 Members eliminated from cluster formation

The Fig 4 shows simulation results of controlling size of cluster. Here all the dark colored nodes are eliminated from cluster formation to control unnecessary size of cluster. After selecting cluster head nodes sends accept message to selected cluster head, with this message nodes also sends control information such as residential energy to the respective $\mathrm{CH}$. This minimizes time required to construct routing tree and minimizes delay in the network for each round.

Algorithm for cluster joining phase

Step 1: Each cluster head sends join request to its members.

Step 2: All members finds signal strength coming from cluster head.

Step 3: Nodes selects cluster head close to it and having maximum signal strength

Step 4: If signal strength is weak then node will not become member of cluster to reduce size of cluster.

\subsubsection{Phase 4 : Cluster tree creation and actual data communication.}

Now each cluster head selects node closer to the base station by calculating weight of all neighboring nodes from following weight vector.

$$
\mathrm{W}=\mathrm{W} 1, \mathrm{~W} 2, \mathrm{~W} 3, \mathrm{WN}
$$

Dijkstras shortest path algorithm is good for finding the shortest path but it only considers distance while selecting the shortest distance .But in WSN with distance energy of node must be considered while selecting parent node[11] Here weight for each node is calculated based on distance and energy. Here optimum path is selected between cluster head and base station. In this way from each cluster head optimum path is selected to the base station .it provides tree like structure and we can say it as routing tree.

Finally Sensor nodes sense data and creates DATA packets and forwards this DATA packet to cluster head only within its time slot provided by cluster head. Energy required for sensing can be calculated by using following equation.

$$
\mathrm{E}_{\mathrm{S}}=\mathrm{Z} * \mathrm{P}_{\mathrm{Si}} * \mathrm{t} \text {. }
$$

Here $\mathrm{P}_{\mathrm{Si}}$ is the size of sensed data.t is the time required for sensing and $\mathrm{Z}$ is the constant.

Now cluster head performs processing such as data aggregation and forwards data without any redundant information by using routing tree created based on weight value .Energy required for communication and reception of $\mathrm{k}$ bit of data over the distance $\mathrm{d}$ can be calculated as

Energy required for communication of data

$$
\operatorname{ET}(\mathrm{k}, \mathrm{d})=\left(\mathrm{E}_{\text {elec }} \times \mathrm{k}\right)+\left(\varepsilon_{\text {amp }} \times \mathrm{k} \times \mathrm{d} 2\right)
$$

Energy required for reseption of data 
Dr Syeda Gauhar Fatima, Syeda Kausar Fatima, Dr K.Anitha Sheela and Syed Shaker Hussaini

$$
\mathrm{ER}(\mathrm{k})=\left(\mathrm{E}_{\text {elec }} \times \mathrm{k}\right)
$$

Here Energy consumed by circuit of transmitter or receiver is denoted by $E_{\text {elec }}$ and 'Energy consumed by amplifier denoted by $\varepsilon_{\text {amp }}$.

\section{SIMULATION AND RESULTS}

Protocol provided in this work performs better load balancing than existing tree based routing protocol in wireless sensor network application. By using this new protocol we can avoid early death of nodes close to the base station. This protocol distributes load on the network evenly and provides proper load balancing and energy efficiency to improve life of WSN.Table2 shows parameters used during implementation of the system.

Table 1 Simulation Parameters

\begin{tabular}{|c|c|c|c|}
\hline Simulation Parameters & Value & $\begin{array}{c}\text { Simulation } \\
\text { Parameters }\end{array}$ & Value \\
\hline No of Sensor Nodes & 100 to 400 & Packet size & 2000 bits \\
\hline Initial Energy of Each Node & $0.25 \mathrm{~J} / \mathrm{node}$ & Traffic Mode & $100 \mathrm{~m} \mathrm{X} 100 \mathrm{~m}$ \\
\hline Electronics Energy Consumption & $50 \mathrm{n} \mathrm{J} / \mathrm{bit}$ & Area of sensing field & $(50 \mathrm{~m}, 150 \mathrm{~m}$ \\
\hline Amplifier transmitting energy & $100 \mathrm{pj} / \mathrm{bit} / \mathrm{m} 2$ & Base station location & \\
\hline $\begin{array}{c}\text { Energy consumption for } \\
\text { aggregation }\end{array}$ & \multicolumn{3}{|c|}{$5 \mathrm{~nJ} / \mathrm{bit} / \mathrm{signal}$} \\
\hline
\end{tabular}

Our NS2 simulation results shows that our system has better performance than existing tree based routing protocols such as GSTEB protocol in terms of energy efficient routing and load balancing. We can see it by using following graphs. Here LCJM is a short name given to our protocol i.e. Location Based Cluster Joining Method fig 5 shows comparison for total energy consumption per round, from the figure it is clear that total energy consumption of proposed work is less as compared to GSTEB and figure 6 shows graph for number of dead nodes per round. Here after 100 rounds GSTEB has 9 dead nodes but proposed work has only 3 dead nodes. Graphs using simulation result shows that in same network conditions and by using same parameters used by GSTEB proposed work performs better

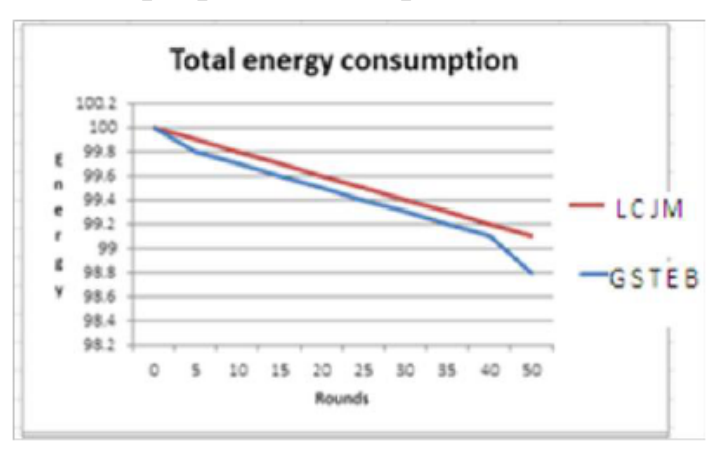

Figure 5 Total energy consumption per round

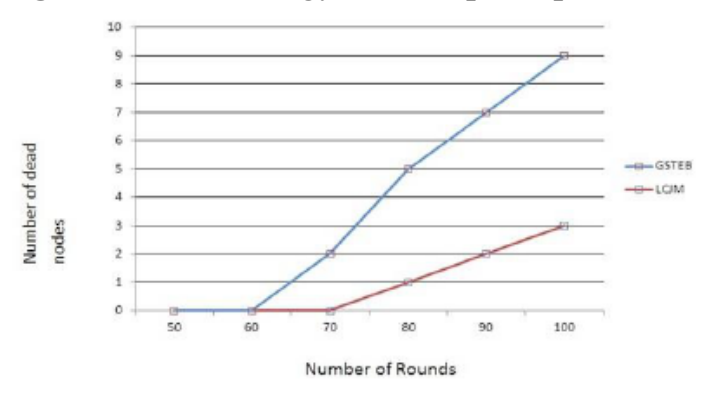

Figure 6 Number of rounds vs. number of dead nodes 


\section{CONCLUSION}

This protocol is different than previous tree based and simple cluster based systems because cluster heads are selected close to the base station to minimize energy consumption due to communication of data from cluster head to the base station .Location based cluster joining method is used to control unnecessary size of cluster to minimize energy consumption. Process of cluster formation starts only after availability of data So these feature provides better load balancing for energy efficient routing to improve life of the network

\section{ACKNOWLEDGMENT}

This work is supported by K.J.College of Engineering and Management Research .I would like to thank my project guide and P.G coordinator for proper guidelines. I would also like to thank all other staff members of my college for their support.

\section{REFERENCES}

[1] Min Yao, Chuang Lin, Yuan Tian "Energy and Delay Minimization in Cluster-Based Wireless Sensor Networks", International Conference on Green Computing and Communications, IEEE 2012.

[2] Yun Li, KokKeong Chai, Yue Chen "Optimal Communication Policy with Joint Minimization on Energy Consumption and Delay for Wireless Sensor Networks", ICCT,IEEE 2013

[3] NabeelaAnsari , Raj Kumar Paul,“ Modified Leach in Wireless Sensor Network”, IOSR Journal of Computer Engineering (IOSR-JCE), Nov - Dec. 2014.

[4] S. Anbuchelian; K. Selvamani; A. Chandarasekar, "An energy efficient multipath routing scheme by preventing threats in Wireless Sensor Networks", Electrical and Computer Engineering (CCECE), IEEE 2014

[5] Supantha Das, Indrajit Banerjee, MainakChatterjee and TuhinaSamanta ,"Performance analysis of TDMA Based Data Communication in WSN",IEEE 2014

[6] CherifaBoucetta; HanenIdoudi; Leila AzouzSaidane, "Ant Colony Optimization based hierarchical data dissemination in WSN", International Wireless Communications and Mobile Computing Conference (IWCMC).IEEE 2015

[7] Wen-Hwa Liao, Yi-An Yen, and Ssu-Chi Kuai "An Efficient Load Balance Data Collection Scheme in Wireless Sensor Networks",IEEE 2015

[8] Ashutosh Bhatia, R. C. Hansdah ,"RD-TDMA: A Randomized and Distributed TDMA Scheduling for Correlated Contention in WSNs",Advanced Information Networking and Applications Workshops (WAINA) Victoria BC May 2014

[9] Zhao Han, Jie Wu, Member, IEEE, Jie Zhang, Liefeng Liu, and KaiyunTian,“A General Self-Organized Tree-Based Energy-Balance Routing Protocol for Wireless Sensor Network", Nuclear Science, IEEE Transactions ,IEE April 2014

[10] M.SENGALIAPPAN, Dr.A.marimuthu "improved general self-organized tree-based routing protocol for wireless sensor network", journal of Theoretical and Applied Information Technology,OCTOMBER 2014.

[11] Sohini Roy, Ayan Kumar Das, "Energy Efficient Cluster Based Routing Protocol (EECBRP) for Wireless Sensor Network ",IEEE 2014.

[12] S. PrabhavathiDr.A. AnandaRaoDr.A.Subramanyam "Globular Topology of Large Scale WSN for Efficient Load Balancing Using Multiple Sink Node", Computing, Communications and Networking Technologies (ICCCNT) ,Fourth International Conference IEEE,2013

[13] Zouhair A. Sadouq; Marouane El Mabrouk; Mohamed Essaaidi, "Conserving energy in WSN through clustering and power control", Third IEEE International Colloquium in Information Science and Technology (CIST),2014.

[14] Priyanka M. Lokhande* and A. P. Thakare "Maximization of Lifetime and Minimization of Delay for Performance Enhancement of WSN", nternational Journal of Business Research and Development Vol. 4 No. 3, pp. 9-16,2015 
Dr Syeda Gauhar Fatima, Syeda Kausar Fatima, Dr K.Anitha Sheela and Syed Shaker Hussaini

[15] PunyashaChatterjee ,Nabanita Das "Multiple Sink Deployment in Multi-Hop Wireless Sensor Networks to Enhance Lifetime", Conference on Applications and Innovations in Mobile Computing (AIMoC),IEE,2015.

[16] Trong-Thua Huynh; Anh-Vu Dinh-Duc; Cong-Hung Tran, "Delay constrained energyefficient cluster-based multi-hop routing inwireless sensor networks",Journal of Communications and Networks Volume: 18, Issue: 4,IEEE 2016.

[17] RanjanDasgupta; Ritwick Mukherjee; Amitava Gupta, "Congestion avoidance topology in wireless sensor network using Karnaughmap", Confarance on Applications and Innovations in Mobile Computing (AIMoC),IEEE, 2015.

[18] ChirihaneGherbi; ZiboudaAliouat; Mohammed Benmohammed, "Distributed energy efficient adaptive clustering protocol with data gathering for large scale wireless sensor networks",conference on Programming and Systems (ISPS), IEEE,2015.

[19] NabajyotiMazumdar, Han Om "An Energy efficient GA-based algorithm for clustering in wireless sensor networks", International Conference on Emerging Trends in Engineering, Technology and Science (ICETETS), ,IEEE, Feb 2016.

[20] KaushikGotefode, KishorKolhe "Energy Efficiency in Wireless Sensor Network using Fuzzy rule and Tree Based Routing Protocol", International Conference on Energy Systems and Applications, Pune,IEEE 30 oct to 1 nov 2015.

[21] VipinPariharParulKansal," Quadrant based routing protocol For improving network lifetime For WSN"'India Conference(INDICON),IEEE, 17 to 20 December 2015

[22] Acharya Mitali Nilesh and Chirag A. Patel, Load Balancing in Cloud Computing Using ant Colony Optimization. International Journal of Computer Engineering \& Technology, 8(6), 2017, pp. 54-59.

[23] Dr. Narayan A. Joshi, Load Balancing in Cloud Using Process Migration, International Journal of Advanced Research in Engineering and Technology (IJARET), Volume 5, Issue 4, April (2014), pp. 230-238

[24] B. Phani Ranga Raja and K. Jyothi Sree, Load Balancing of Feeder Using Fuzzy and Optimization Technique: International Journal of Electrical Engineering \& Technology, 9(4), 2018, pp. 74-82

[25] Ramesh A, Raghunadha Reddy T, Neeraja K, Load Balancing Technique in Cloud Computing Environment, International Journal of Mechanical Engineering and Technology 8(12), 2017, pp. 561-568

[26] T. N. Anitha and Dr. Balakrishna. R, Load Balancing - Server Availability Issue, International Journal of Computer Engineering and Technology (IJCET), Volume 5, Issue 8, August (2014), pp. 71-79

[27] Soumen Swarnakar, Ritika Kumari, Tripti Sinha, Ritika Chakraborty and Tanushree Sarkar, Modified Load Balancing Strategy Based on Cloud Partitioning Status. International Journal of Advanced Research in Engineering and Technology, 9(3), 2018, pp. 17-24.

[28] Vishal A. Nawale and Prof. Priya Deshpande, Survey on Load Balancing and Data Skew Mitigation in Mapreduce Applications, International Journal of Computer Engineering and Technology (IJCET), Volume 6, Issue 1, January (2015), pp. 32-41

\section{ABOUT AUTHOR}

First Author Completing his Master of Engineering at K.J College of engineering and management research, Pune University. He has completed Bachelor of Engineering from P.V.P.I.T, Shivaji University, Kolhapur.

Second Author Working as assistant professor and head of department at Computer Engineering department at KJCOEMR Pune, savitribaiPhule University. He has 10 years teaching experience. He has completed $\mathrm{M}$ Tech in computer science and engineering and $\mathrm{PhD}-$ Pursuing at sathyabama university, Chennai. 